\title{
A huge pancreatic mass
}

\section{Guido Poggia, Benedetta Montagnaa, Chiara Picchia, Claudio Clemente ${ }^{b}$}

Institute of Care Città di Pavia, Pavia; Policlinico San Donato, Milano, Italy

An 82-year-old woman with a history of dyspepsia, early satiety and an intra-abdominal mass, known for many years and thought to be a cyst, presented with jaundice. Physical examination revealed a right upper abdominal mass and scleral icterus. Abdominal computed tomography found a well demarcated, 15-cm mass of the pancreatic head, enhanced during the arterial phase of contrast medium, causing compression and distension of the biliary tree (Fig. 1). Histological sampling showed solid areas composed of irregular pseudopapillary structures (Fig. 2). Immunohistochemical stains were positive for vimentin, cytokeratin and progesterone and negative for trypsin and chymotrypsin. Solid pseudopapillary tumors of the pancreas are rare exocrine pancreatic tumors occurring primarily in young women and occasionally in the elderly. They usually exhibit an indolent biological behavior. Surgical resection is the treatment of choice [1]. The patient refused duodenopancreatectomy therefore a biliary stent was placed with complete jaundice disappearance.

\section{Reference}

1. Zhang H, Liang TB, Wang WL, Shen Y, Ren GP, Zheng SS. Diagnosis and treatment of solid-pseudopapillary tumor of the pancreas. Hepatobiliary Pancreat Dis Int 2006;5:454-458.

${ }^{\text {a} O n c o l o g y ~ U n i t, ~ I n s t i t u t e ~ o f ~ C a r e ~ C i t t a ̀ ~ d i ~ P a v i a, ~ P a v i a ~(G u i d o ~ P o g g i, ~}$

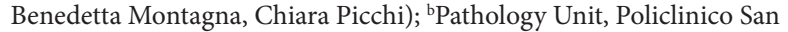
Donato, Milano (Claudio Clemente), Italy

\section{Conflict of Interest: None}

Correspondence to: Guido Poggi, MD, Chief, Oncology and Hepatology Unit, Istituto di Cura Città di Pavia, 27100 Pavia, Italy, Tel.: +39 0382433631, Fax: +39 0382576821,

e-mail: guidopoggi64@gmail.com, guido.poggi@grupposandonato.it

Received 27 May 2016; accepted 26 May 2016; published online 24 June 2016

DOI: http://dx.doi.org/10.20524/aog.2016.0068

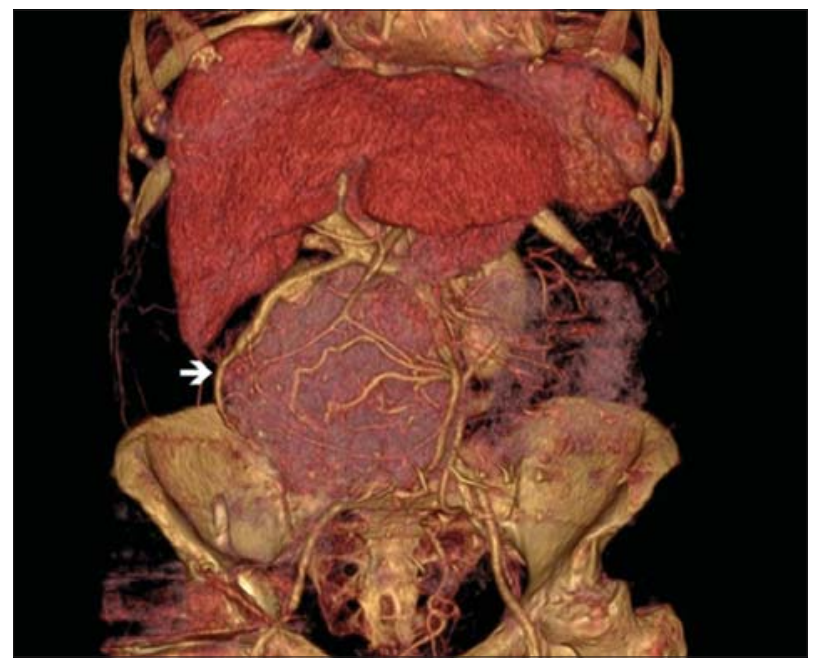

Figure 1 A 15-cm mass of the pancreatic head (white arrow)

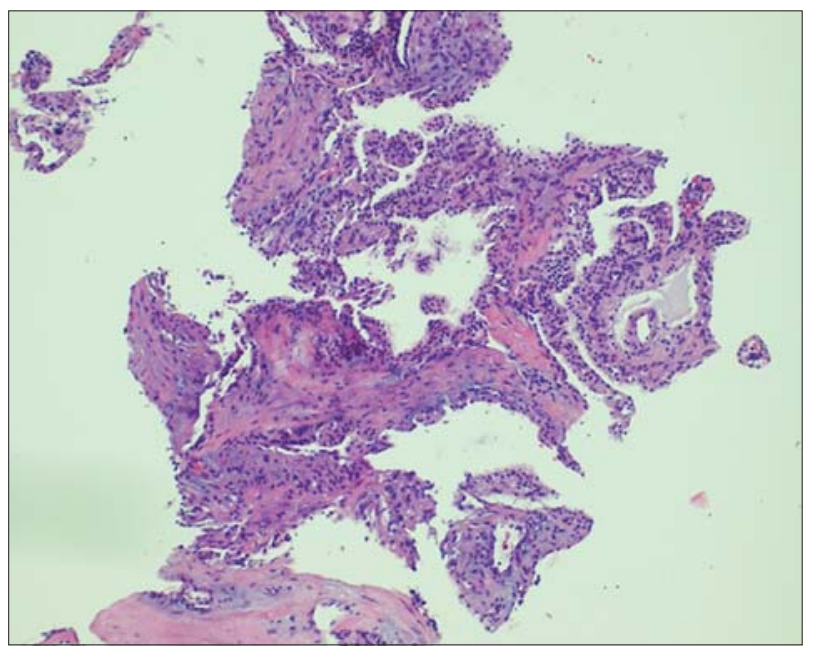

Figure 2 Histological sampling (HE x 10) showed solid areas composed of irregular pseudopapillary structures 